Check for updates

Cite this: Mater. Chem. Front. 2020, 4, 128

\section{Förster resonance energy transfer (FRET) paired carbon dot-based complex nanoprobes: versatile platforms for sensing and imaging applications}

\author{
Shihai Miao, ${ }^{a}$ Kang Liang (DD ${ }^{\text {bc }}$ and Biao Kong (D) *a
}

\begin{abstract}
As one of the most promising carbon-based photoluminescent materials, carbon dots (CDots) have recently attracted great attention for many potential applications owing to their excellent optical, electrical, and chemical properties. Förster Resonance Energy Transfer (FRET) is a highly sensitive spectroscopic technique that has been widely utilized for all applications of fluorescence. In this review, we provide an updated roadmap of CDot-based FRET systems for nanoprobes. Several CDot-based FRET systems obtained by co-assembling different fluorescent molecules are surveyed. Furthermore, the synthesis strategies for CDot-based FRET systems and their applications in nanoprobes, bioimaging, and biomedicine are reviewed. We further provide an outlook toward the future development of CDot-based FRET systems. This article summarizes the latest progress in CDot-based FRET systems and looks forward to further developments in these exciting nanocomposites.
\end{abstract}

Received 25th August 2019,
Accepted 3rd October 2019

DOI: $10.1039 / c 9 q m 00538 b$

rsc.li/frontiers-materials

\footnotetext{
${ }^{a}$ Department of Chemistry, Collaborative Innovation Center of Chemistry for Energy Materials (iChEM), Laboratory of Advanced Materials, Shanghai Key Laboratory of Molecular Catalysis and Innovative Materials, Fudan University, Shanghai 200433, P. R. China. E-mail: bkong@fudan.edu.cn; Web: https://softnanolab. fudan.edu.cn; Fax: +86-21-31249176; Tel: +86-21-31249176

${ }^{b}$ School of Chemical Engineering, Graduate School of Biomedical Engineering, The University of New South Wales, Sydney NSW 2052, Australia ${ }^{c}$ Australian Centre for NanoMedicine, The University of New South Wales, Sydney NSW 2052, Australia
}

\section{Introduction}

Förster resonance energy transfer (FRET) is the mechanism by which non-radiative energy is transferred between a luminescent donor and an energy acceptor within a close range of approximately 10 to $100 \AA^{1-3}{ }^{1-3}$ FRET can be easily measured in standard bulk fluorescence experiments. FRET can also be monitored using fluorescence microscopy techniques, including single-molecule fluorescence spectroscopy. FRET serves as a highly sensitive spectroscopic technique that has been widely utilized in all applications of fluorescence, including medical

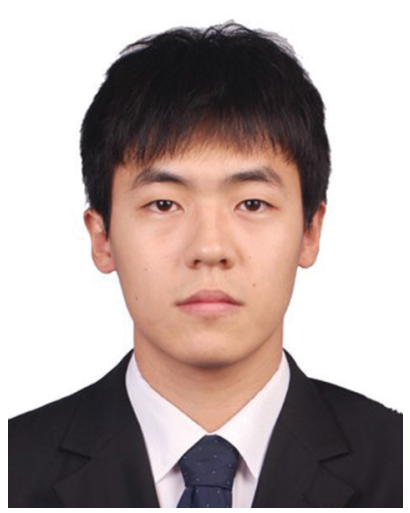

Shihai Miao
Shihai Miao obtained a bachelor's degree (2013) in Materials Chemistry at China University of Geosciences (Beijing) and a master's degree (2016) in Materials Engineering at China University of Geosciences (Beijing). He is currently an assistant researcher under the supervision of Prof. Dr Biao Kong in the Soft Nano Group at Fudan University. His research is focused on the design, synthesis, and characterization of carbon nanodots for applications in biosensing and flexible display devices.

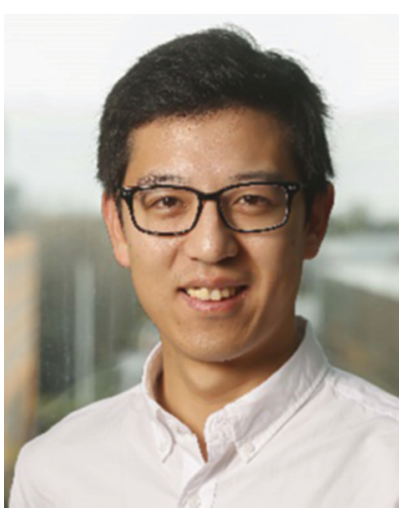

Kang Liang
Dr Kang Liang is a specially invited researcher and Scientia Fellow in the department of Chemical Engineering and Biomedical Engineering at The University of New South Wales. He received a Bachelor of Engineering degree with Honors (Biomedical) in 2009 from the University of Melbourne (Australia) and commenced his $P h D$ in 2010 under the supervision of Professor Frank Caruso. His research interests are primarily focused on the application of advanced bio-nano interface materials in biomedicine. 
diagnosis, biological analysis, and optical imaging. ${ }^{4-7}$ FRETbased dual-emission nanoprobes for ratiometric fluorescence sensing are achieved by synthesizing nanoparticles from two or more fluorescent molecules with different emission bands. One fluorophore serves as the reference, while the other acts as a response molecule, allowing the FRET system to be used as a ratiometric fluorescence nanoprobe. To date, many excellent studies and reviews of FRET have been conducted. In 2013, Chen et al. reviewed fluorescent nanosensors based on FRET. This review introduced the design and applications of sensitive and selective FRET-based ratiometric nanoprobes in detail. This review also classified nanosensors based on the type of nanoparticle and demonstrated the design and application of FRET-based fluorescent nanosensors for the detection of metal ions, small molecules, DNA, and other analytes. ${ }^{17}$ In 2014, Prevo et al. reviewed FRET and kinesin motor proteins. The physical basis of FRET and how to apply FRET to biological molecules was systematically introduced. Prevo et al. focused on the application of different FRET methods in motor protein drivers that had undergone several conformational changes under the action of enzymes. This FRET system moves unidirectionally along the microtubule filament, driving active intracellular transport. ${ }^{18}$ In 2014, Algar et al. examined the applications of FRET systems based on semi-conductor quantum dots. They reviewed the characterization of nontraditional structures of quantum dot-based FRET systems and their applications in biosensors, energy conversion, and optoelectronic device manufacturing in recent years. ${ }^{19}$ Although many applications of FRET have been reported, it is still an analytical tool that must continue to be developed. For example, the study of new energy-transfer donor and receptor pairs in the FRET system is important to improve FRET efficiency and analytical performance.

Carbon dots (CDots) are newly emerging carbon-based fluorescent nanomaterials that have received considerable

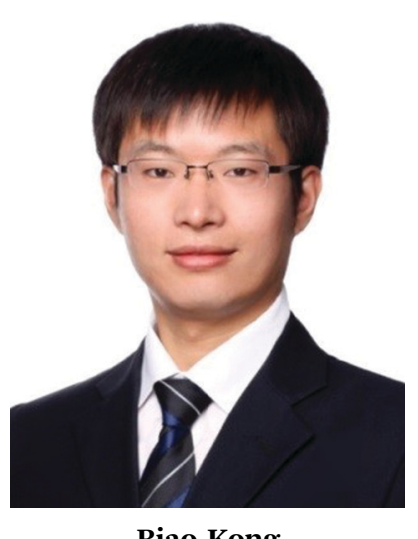

Dr Biao Kong is currently a professor and National Thousand Youth Talents Plan fellow at Fudan University. He completed $\mathrm{PhD}$ degrees from Monash University (Australia) and Fudan University (China) under the supervision of Prof. Dongyuan Zhao and Selomulya Cordelia in 2015. He then served as a Research Fellow at the University of Melbourne (Australia) in 2015 and a Postdoctoral Fellow at Stanford University (USA) in 2016. Subsequently, he was appointed as a professor of Global Expert of Thousand Talents Plan at Fudan University. His scientific interests focus on the rational design and fabrication of super-assembled framework-based soft heterointerfaces and integrated devices for energy, catalysis, and biological applications. attention in recent years. Owing to their high optical stability, ease of synthesis and surface functionalization, good biocompatibility, and adjustable composition, CDots exhibit great potential in various applications such as catalysis, nanoprobes, optoelectronic devices, bioimaging, and biomedicine. ${ }^{20-25}$ The first discovery of carbon-based fluorescent nanomaterials was in 2004, when $\mathrm{Xu}$ et al. synthesized single-walled carbon nanotubes via an electrophoretic and purification process using arc soot as a raw material. Surprisingly, fluorescent carbon was found separate from the mixture of reactants. ${ }^{26}$ The first report of CDots as a carbon-based fluorescent nanomaterial was in 2006, when Sun et al. synthesized quantum-sized CDots through the laser ablation of a carbon target in the presence of water vapor using argon as a carrier gas. As the excitation wavelength increased, the emission wavelength of the CDots showed a continuous red shift. ${ }^{27}$ Since then, CDot research and reports have expanded rapidly. In 2012, Wei et al. showed the possibility and importance of CDots as energy donors to build FRET systems for the first time. The fluorescence of CDots can be quenched by graphene via the FRET process and then restored upon the introduction of $\mathrm{K}^{+} .^{28}$ In 2013, Tang et al. reported an efficient CDot-based FRET system for the real-time monitoring of drug delivery and bioimaging. In this system, CDots served as both a FRET donor and a drug carrier, and doxorubicin served as an acceptor. ${ }^{9}$ In 2016, Yu et al. reported FRET between CDots and rhodamine B based on an up-conversion process spanning three components, resulting in the efficient utilization of the apparently wasted portion of sunlight (200-800 nm). ${ }^{29}$ In 2019, Su et al. described a straightforward strategy for preparing nanocomposites via the co-assembly of CDots and boron dipyrromethene based on FRET. The nanocomposite exhibited high water solubility, good biocompatibility, and photodynamic therapy efficiency. ${ }^{30}$

Many research efforts have attempted to build Cdot-based FRET systems as nanoprobes. Fig. 1 shows a timeline highlighting recent research efforts on Cdot-based FRET systems. ${ }^{8-16}$ Many fluorescent molecules can be employed to construct FRET systems with CDots. Generally, a FRET system contains two kinds of fluorescent molecules with different emission wavelengths. When CDots act as donors, they transfer energy to another molecule to construct the FRET system. Conversely, when the CDots act as receptors, they accept energy from other molecules, achieving ratiometric luminescence via FRET. Previous reports demonstrate that paired CDot-based FRET systems exhibit high sensitivity and have been widely utilized in all fluorescence-based applications, including medical diagnosis, biological analysis, and optical imaging. As CDots and FRET have been detailed in many excellent reviews, this review focuses on Cdot-based FRET systems with respect to their construction strategies, beneficial properties, and potential in a variety of applications.

In this review, we present details on several Cdot-based FRET systems co-assembled from different classes of fluorescent materials, including organic fluorescent molecules, drug molecules, metal complexes, and nanomaterials. Based on the different fluorescent molecules, different combinations can be used to construct hetero-interfaces with CDots. These hetero-interfaces 




Fig. 1 Timeline showing recent studies on CDot-based FRET systems. ${ }^{8-16}$ Reprinted with permission ref. 9-17. Copyright 2012 Wiley, Copyright 2013 Wiley, Copyright 2014 Wiley, Copyright 2015 The Royal Society of Chemistry, Copyright 2016 John Wiley and Sons Ltd, Copyright 2016 American Chemical Society, Copyright 2017 American Chemical Society, Copyright 2018 Elsevier, Copyright 2019 Elsevier.

are mainly based on covalent binding and surface functionalization/coupling, thus resulting in different FRET effects. We also review the synthetic strategies for Cdot-based FRET systems and the applications of these systems in nanoprobes, bioimaging, and biomedicine.

\section{Nanoprobes based on CDots and fluorescent molecules}

\subsection{CDots \& fluorescent molecules}

Fluorescent molecules exhibit characteristic fluorescence in the ultraviolet-visible-near-infrared region, and their fluorescence properties (excitation and emission wavelengths, intensity, lifetime, polarization, etc.) can be sensitively changed based on the properties of the environment (e.g., polarity, refractive index, and viscosity). Based on the excellent and diverse properties of fluorescent molecules, many promising applications have been demonstrated. Unsurprisingly, many studies have focused on FRET between CDots and fluorescent molecules. Table 1 presents a survey of recent works on FRET systems based on fluorescent molecules and CDots along with their applications.

In 2012, Huang et al. reported a CDot-chlorin e6 FRET system based on a theranostic platform. Fig. 2a shows the FRET process between the CDots and chlorin Ce6, which combined through covalent binding. FRET occurs from the CDots to chlorin e6, and the CDots and CDots-chlorin e6 displayed green and red fluorescence, respectively. The CDots-Ce6 FRET system shows promise for applications in near-infrared fluorescence imagingguided photodynamic therapy due to its excellent imaging and tumor-homing properties. $^{8}$ In 2013, Du et al. synthesized a ratiometric fluorescent nanoprobe with low cytotoxicity and high reversibility based on FRET for $\mathrm{pH}$ sensing (Fig. 2b).
In this nanoprobe, fluorescein isothiocyanate was assembled onto the CDots, which acted as the energy-transfer donor and carrier. The nanoprobe was successfully used to image live cells and reveal intracellular pH gradients. ${ }^{33}$ In 2014, Du et al. reported a FRET-based ratiometric nanoprobe for mitochondrial $\mathrm{H}_{2} \mathrm{O}_{2}$ in living cells (Fig. 2c). The FRET from the CDots (donor) to boronate-protected fluorescein (PF1; acceptor), which is covalently linked to the CDots, results the blue emission of the CDots. As $\mathrm{H}_{2} \mathrm{O}_{2}$ is added, FRET occurs between the CDots and PF1, resulting in green fluorescence. The, the ratiometric detection of $\mathrm{H}_{2} \mathrm{O}_{2}$ is realized. ${ }^{10}$ In 2018, Chatzimarkou et al. synthesized a sensitive and selective FRET-based nanoprobe for 4-nitrophenol (Fig. 2d). The synthesized N-CDots exhibited blue fluorescence, which can effectively be quenched by 4-nitrophenol through the FRET process from CDots to 4-nitrophenol. The CDots/4-nitrophenol showed bright luminescence and low toxicity, demonstrating promise for cell imaging and other biosensing applications. ${ }^{15}$

\subsection{CDots \& drug molecules}

Drug molecules play a vital role in processes related to human health, such as nucleic acid repair, cell apoptosis, and tumor suppression. When coupled with CDots, certain drugs can be utilized to construct CDot-drug FRET systems, which show promising applications in nanoprobes, bioimaging, and biomedicine. Drug molecules commonly used in FRET include doxorubicin, ${ }^{9,56,57}$ porphyrins,${ }^{58,59}$ vitamins,${ }^{53,55,60}$ curcumin, ${ }^{61}$ rhodizonate, ${ }^{62}$ and cisplatin(Iv) prodrug. ${ }^{54}$

In 2013, Tang et al. reported an efficient CDot-based FRET system for the real-time monitoring of drug delivery and bioimaging. Fig. 3a shows the assembly process of a FRETCDots drug delivery system (DDS) via direct surface coupling and the proposed mechanism of the FRET-CDots DDS for drug delivery. In this system, the CDots serve as both a donor and a 
Table 1 Representative examples of FRET systems based on fluorescent molecules and CDots along with their applications

\begin{tabular}{|c|c|c|c|c|c|}
\hline Coupling molecule & Application & Ref. & Coupling molecule & Application & Ref. \\
\hline Naphthalimide & NO nanoprobe & 32 & Isothiocyanate & PH nanoprobe & 33 \\
\hline NBD-PE; BODIPY-PH & Bioimaging & 34 & Rhodamine B & $\mathrm{Fe}^{3+}$ nanoprobe & 35 \\
\hline Nitroaromatics & Nanosensor & 13 & NBD-PE & Nanoprobe, bioimaging & 37 \\
\hline Rhodamine B & Up-conversion properties & 29 & Chlorin e6 & Bioimaging & 38 \\
\hline Rhodamine 6G & $\mathrm{Fe}^{3+}$ nanoprobe & 39 & $\mathrm{TNB}^{-}$ & DDVP nanoprobe & 40 \\
\hline Fluorescein & $\mathrm{NH}_{3}$ nanoprobe & 45 & Isothiocyanate & PH nanoprobe & 46 \\
\hline Acridone derivate & RNA nanoprobe & 47 & Naphthalimide & Tyrosinase nanoprobe & 48 \\
\hline Rhodamine123 & Bioimaging & 49 & Nitrophenol & Nanoprobe, bioimaging & 15 \\
\hline Acriflavine & SEB nanoprobe & 50 & Fluorescein (FAM) & $\mathrm{Zn}^{+}$nanoprobe, bioimaging & 51 \\
\hline Boron dipyrromethene & Bioimaging & 30 & Naphthalimide & Cys nanoprobe, bioimaging & 52 \\
\hline
\end{tabular}

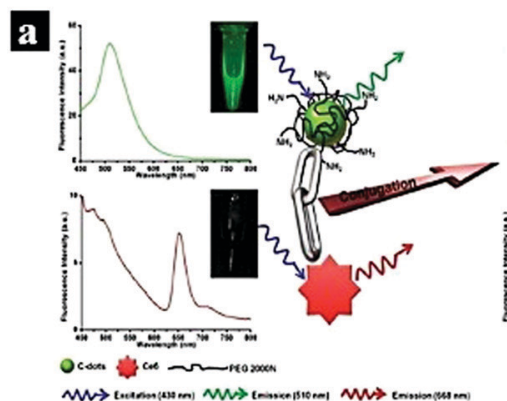

c

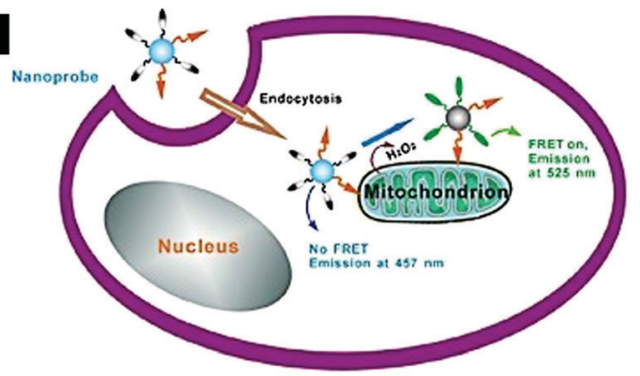

:Carbon dot
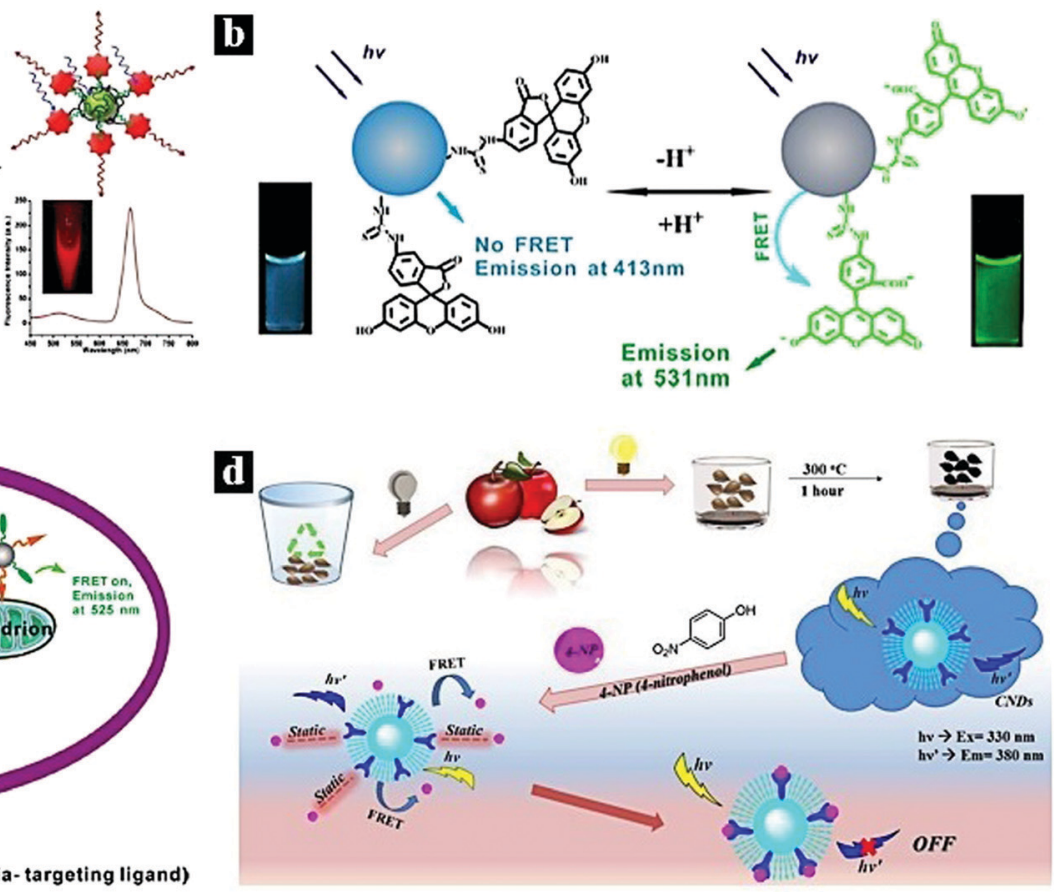

at $531 \mathrm{~nm}$

Fig. 2 (a) FRET process between CDots and chlorin e6. ${ }^{8}$ Reprinted with permission ref. 8. Copyright 2012 Wiley. (b) Schematic illustration of a Cdot-based FRET nanoprobe for $\mathrm{pH}$ sensing. ${ }^{33}$ Reprinted with permission ref. 33. Copyright 2013 IOP Publishing Ltd. (c) Nanoprobe using FRET-based $\mathrm{H}_{2} \mathrm{O}_{2}$ ratiometric sensing in a living cell. ${ }^{10}$ Reprinted with permission ref. 10. Copyright 2014 Wiley-VCH Verlag. (d) Schematic illustration of the synthetic process of CDots and the FRET process between CDots and 4-nitrophenol. ${ }^{15}$ Reprinted with permission ref. 15. Copyright 2018 Elsevier.

drug carrier, and doxorubicin serves as an acceptor. As doxorubicin is released from the surfaces of the CDots, the FRET between doxorubicin and the CDots is terminated, restoring the fluorescence of the CDots. ${ }^{9}$ In 2015, Wang et al. reported a Cdot-based FRET system with high quantum yield (QY) for the detection of biologically significant vitamin $B_{12}$. Fig. 3 b shows the thermal reduction process of the high-QY CDots and the FRET process between the CDots and vitamin $B_{12}$. The low-QY CDots exhibited emission wavelengths at $445 \mathrm{~nm}$; after thermal treatment, the CDots showed high QY with an emission wavelength of $431 \mathrm{~nm}$. The CDots were used to detect vitamin $B_{12}$ based on the FRET between the CDots and vitamin $\mathrm{B}_{12} \cdot{ }^{53}$ In 2017, Feng et al. reported a CDot-based system for the real-time ratiometric monitoring of anticancer prodrug activation in living cells.
Fig. 3c shows the FRET process between the CDots and cisplatin(Iv) prodrug. The quencher unit attached to the CDot surfaces through amide condensation. The CDots showed blue, green, and red emission under different excitation conditions as a drug nanocarrier. Based on the FRET between the CDots and cisplatin(Iv) prodrug, the blue emission of the CDots was quenched. Under reductive conditions, the nanocarrier released the dabsyl unit and active Pt(II), causing the blue emission of the CDots to recover with time; meanwhile, the intensity of green and red fluorescence remained almost unchanged. ${ }^{54}$ In 2015, Wang et al. reported a ratiometric fluorescent nanoprobe based on a N,S-CDots FRET system that was used for the determination of eriboflavin in aqueous solutions (Fig. 3d). The N,S-CDots exhibited blue emission at 
$\mathbf{a}^{\mathrm{a}}$



CDot

b)

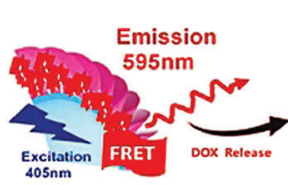

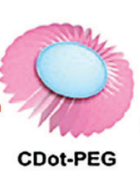
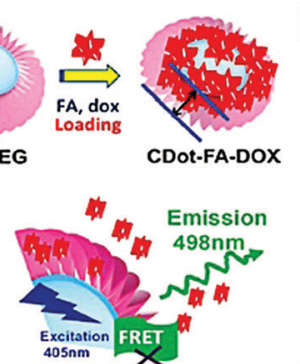

c

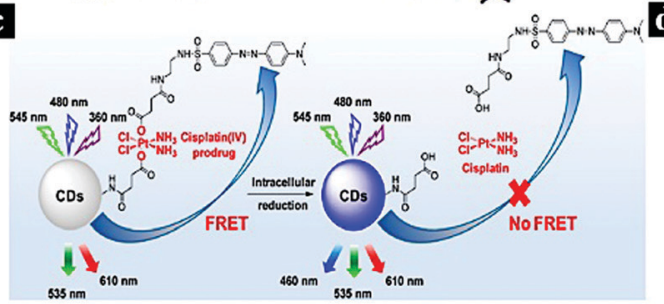

b
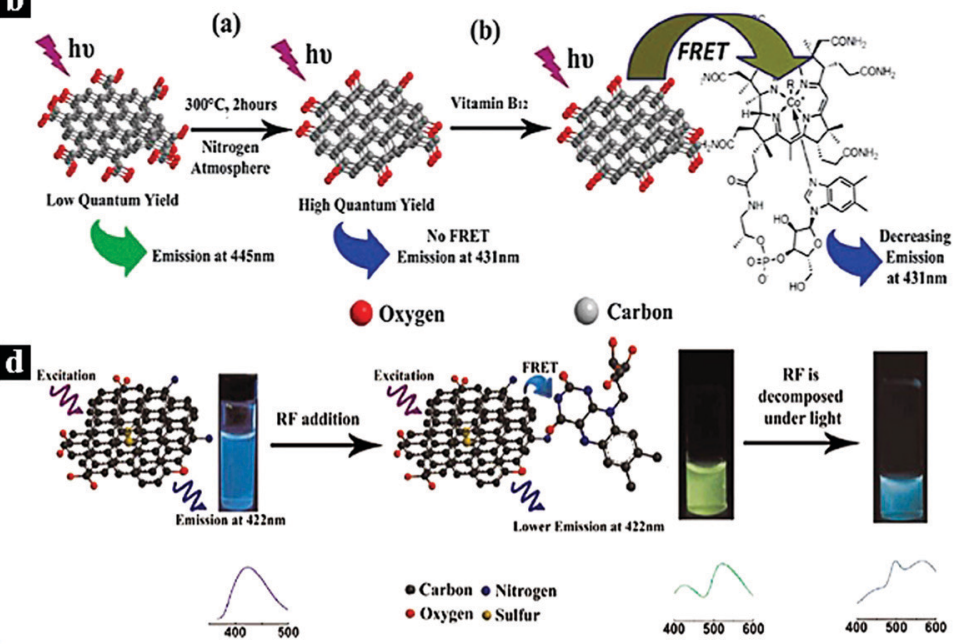

Fig. 3 (a) Assembly process of FRET-CDots-DDS and the proposed mechanism of FRET-CDots-DDS for drug delivery. ${ }^{9}$ Reprinted with permission ref. 9. Copyright 2013 Wiley. (b) Thermal reduction of high-QY CDots and the FRET process between CDots and vitamin $B_{12} .{ }^{53}$ Reprinted with permission ref. 53. Copyright 2015 The Royal Society of Chemistry. (c) FRET process between CDots and cisplatin(IV) prodrug. ${ }^{54}$ Reprinted with permission ref. 54 . Copyright 2017 American Chemical Society. (d) Schematic diagram of a riboflavin probe based on N,S-CDots and the FRET process. ${ }^{55}$ Reprinted with permission ref. 55. Copyright 2015 Elsevier.

$465 \mathrm{~nm}$; through the FRET process from N,S-CDots to riboflavin, the blue emission intensity decreased monotonically, while the green emission intensity increased. ${ }^{55}$

\subsection{CDots \& metal complexes}

The monitoring of metal ions is meaningful in the fields of human health and environmental protection. FRET systems based on CDot-metal complexes can act as efficient and sensitive nanoprobes for the detection of certain metal ions. The most investigated metal complexes include rhodamine- $\mathrm{Al}^{3+},{ }^{63}$ cobalt oxyhydroxide, ${ }^{64,65}$ quercetin- $\mathrm{Zn}^{2+}, 66$ cyclam- $\mathrm{Cu}^{2+},{ }^{27}$ $p$-phenylenediamine- $\mathrm{Cu}^{2+},{ }^{14}$ ruthenium complex, ${ }^{68}$ and $\mathrm{HCM}-\mathrm{Cu}^{2+}{ }^{2}{ }^{9}$

In 2015, Kim et al. designed a CDot-rhodamine-Al ${ }^{3+}$ FRETbased nanoprobe for $\mathrm{Al}^{3+}$ detection in aqueous solution. Fig. $4 \mathrm{a}$ schematically illustrates the FRET process between the CDots and rhodamine- $\mathrm{Al}^{3+}$, which are covalently combined. The CDots act as an energy donor, while rhodamine- $\mathrm{Al}^{3+}$ serves as an energy acceptor in the presence of $\mathrm{Al}^{3+}$ ions. The emission of the CDots can be tuned from blue to green based on the FRET process from the CDots to rhodamine- $\mathrm{Al}^{3+} .{ }^{63}$ In 2017, Liu et al. synthesized dual optical CDots-based ratiometric fluorescent nanoprobe for $\mathrm{Cu}^{2+}$. Fig. $4 \mathrm{~b}$ schematically illustrates the FRET process from the blue CDots to the $p$-phenylenediamine- $\mathrm{Cu}^{2+}$ complex. $\mathrm{As}^{2+}$ can coordinate with - $\mathrm{COOH}$ and $p$-phenylenediamine, this led to blue CDots joining to the surface of red CDots. FRET occurred from the blue CDots to $p$-phenylenediamine- $\mathrm{Cu}^{2+}$ on the surfaces of the red CDots, leading to the fluorescence quenching of the blue CDots. The emission color could be tuned from blue to red based on the FRET process by adjusting the $\mathrm{Cu}^{2+}$ doping content. ${ }^{14}$ In 2015, Yang et al. reported fluorescent CDots as a sensitive nanoprobe for $\mathrm{Zn}^{2+}$. Fig. $4 \mathrm{c}$ schematically illustrates the detection of $\mathrm{Zn}^{2+}$ based on a FRET system comprising CDots and pentahydroxyflavone- $\mathrm{Zn}^{2+}$ complex. Upon excitation at $360 \mathrm{~nm}$, FRET occurred from the CDots to pentahydroxyflavone- $\mathrm{Zn}^{2+}$, while pentahydroxyflavone- $\mathrm{Zn}^{2+}$ accepted the energy and exhibited fluorescence emission at $480 \mathrm{~nm} .{ }^{66}$ In 2018, Yan et al. reported a FRET-based ratiometric fluorescent nanoprobe for $\mathrm{Cu}^{2+}$ using 7-diethylaminocoumarin-3-carbohydrazide ( $\mathrm{CMH}$ )-functionalized CDots (Fig. 4d). In this system, $\mathrm{CMH}$ is covalently bound to the surfaces of the CDots, and FRET occurs from the CDots to CMH. However, the FRET process was inhibited by adding $\mathrm{Cu}^{2+}$; meanwhile, the fluorescence intensity increased at $400 \mathrm{~nm}$ and decreased at $458 \mathrm{~nm}$. The emission can be tuned from blue to blue-green in the presence and absence of $\mathrm{Cu}^{2+}$ under UV light. ${ }^{69}$

\subsection{CDots \& biomolecules}

Biomolecules are an important part of higher flora and fauna due to their indispensable functions, such as helping cells transmit signals and controlling physiological and pathological processes. The biomolecules commonly applied in FRET include nucleic acids, ${ }^{70}$ tryptophan, ${ }^{71}$ and dopamine. ${ }^{72}$

In 2017, Khakbaz et al. reported a nanoprobe for micro-RNA sensing based on a FRET system. The FRET occurs between CDots and carboxyfluorescein-labeled DNA, and the CDot fluorescence is quenched. The nanoprobe can be used for micro-RNA detection and the early detection of breast cancer. ${ }^{70}$ In 2017, Dang et al. synthesized a Cdot-based FRET system via a onestep method using tryptophan and $\beta$-cyclodextrin as precursors. FRET was found to occur between tryptophan and the CDots, and this system could be used as a sensitive probe for $\mathrm{Fe}^{3+}$ detection. ${ }^{71}$ In 2017, Zhang et al. reported a FRET system based on polymerized dopamine (PDA) and CDots for bioimaging (Fig. 5). The FRET effect occurs between the CDots and PDA, resulting in highly efficient and tunable emission. The cell imaging ability and cytotoxicity of the CDot-PDA system were also explored, indicating 


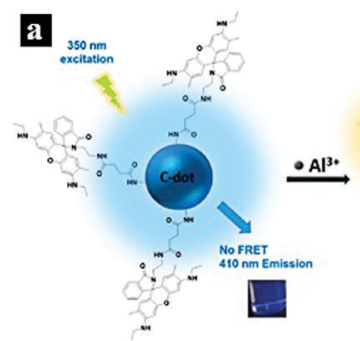

b


Fig. 4 (a) Schematic illustration of the FRET process between CDots and rhodamine-Al ${ }^{3+}{ }^{63}$ Reprinted with permission ref. 63 . Copyright 2015 American Chemical Society. (b) Schematic illustration of the FRET process from blue CDots to $p$-phenylenediamine-Cu ${ }^{2+} \cdot{ }^{14}$ Reprinted with permission ref. 14 . Copyright 2017 American Chemical Society. (c) Schematic illustration of the detection of $\mathrm{Zn}^{2+}$ based on a CDots/pentahydroxyflavone-Zn ${ }^{2+}$ FRET system. ${ }^{66}$ Reprinted with permission ref. 64. Copyright 2015 Springer-Verlag Wien. (d) Schematic illustration of a FRET system based on CDots and $\mathrm{CMH}-\mathrm{Cu}^{2+}{ }^{26}$ Reprinted with permission ref. 65. Copyright 2018 Elsevier.
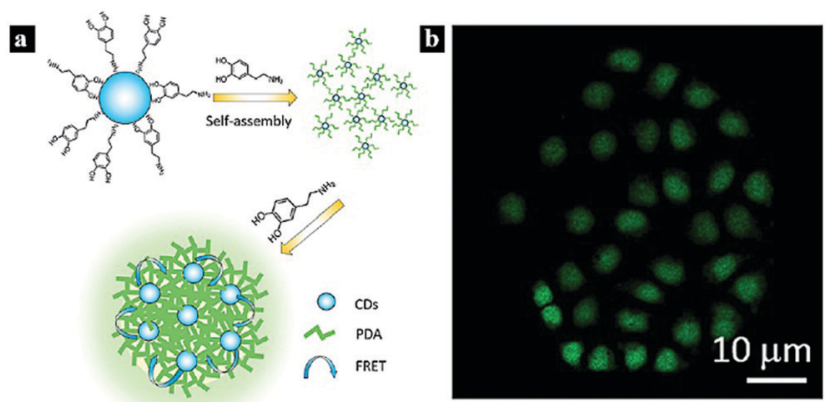

Fig. 5 (a) Schematic illustration of a nanoprobe based on FRET between CDots and PDA. (b) Confocal fluorescence images of MCF-7 cells under $405 \mathrm{~nm}$ laser excitation. Reprinted with permission ref. 76. Copyright 2017 The Royal Society of Chemistry.

good biocompatibility and strong prospects for biotechnological applications. $^{72}$

\section{Nanoprobes based on CDots and nanomaterials}

\subsection{CDots \& $\mathrm{MnO}_{2} / \mathrm{MoS}_{2}$}

Layered transition-metal disulphides or dioxides (e.g., $\mathrm{MnO}_{2}$, $\mathrm{WS}_{2}$, and $\mathrm{MoS}_{2}$ ) are a class of two-dimensional nanomaterials that show excellent properties, including large surface areas along with semiconducting and energy-harvesting characteristics, giving them widespread applications. Because of their excellent optical absorption capability and fast electron transfer rate, these materials are promising fluorescence quenchers and have attracted considerable attention in the construction of FRET-based sensing platforms.

$\mathrm{MnO}_{2}$ is one of the most stable manganese oxides and possesses excellent chemical and physical properties. As a transition metal oxide, it attracts considerable attention in batteries, supercapacitors, and even catalysis driven by visible light. $\mathrm{MnO}_{2}$ shows broad absorption in the range of $250-550 \mathrm{~nm}$, which overlaps with the fluorescence excitation of CDots. This phenomenon allows the occurrence of FRET between CDots and $\mathrm{MnO}_{2} \cdot{ }^{11,73-79}$ The transition metal oxide $\mathrm{MoS}_{2}$ has a sandwich structure that is bonded by weak van der Waals forces and can be regarded as S-Mo-S. $\mathrm{MoS}_{2}$ possesses a large intrinsic band gap that depends largely on the number of layers. In addition, most transition metal ions have inherent fluorescence quenching characteristics. Therefore, many FRET systems based on CDots and $\mathrm{MoS}_{2}$ have been reported. ${ }^{80-83}$

In 2015, Wang et al. reported a FRET system between CDots and $\mathrm{MnO}_{2}$ for glutathione (GSH) sensing in human whole blood samples (Fig. 6a). Due to the FRET from the CDots to $\mathrm{MnO}_{2}$ through $\mathrm{MnO}_{2}$-surface functionalization with CDots, the fluorescence emission of the CDots was quenched. After the introduction of GSH, the fluorescence of the CDots was restored. ${ }^{11}$ In 2018, Yan et al. synthesized a CDots- $\mathrm{MnO}_{2}$ FRET platform and applied it in the sensitive detection of organophosphorus pesticides (Fig. 6b). The fluorescence intensity of the CDots can be tuned off via FRET from CDots to the energy acceptor $\mathrm{MnO}_{2}$. Moreover, the quenching effect induced by $\mathrm{MnO}_{2}$ nanosheets can be restored by adding acetylthiocholine and butyrylcholinesterase to the FRET system. ${ }^{74}$ In 2015, Yang et al. reported a turn-on fluorescence nanoprobe for GSH in aqueous solutions based on a CDots- $\mathrm{MnO}_{2}$ FRET system (Fig. 6c). The CDots- $\mathrm{MnO}_{2}$ complex is easily synthesized via surface functionalization and exhibits a stable FRET process. The MnO can be reduced in the presence of GSH introduced into the system, thereby inhibiting the FRET process between the CDots and $\mathrm{MnO}_{2}$ and restoring the fluorescence intensity. ${ }^{75}$ In 2017, Jana et al. reported a CDots- $\mathrm{MnO}_{2}$ FRET system for molecular logic operations using $N$-acetyl-L-cysteine (NAC) and $\mathrm{H}^{+}$as inputs (Fig. 6d). The FRET process occurs from the CDots to 

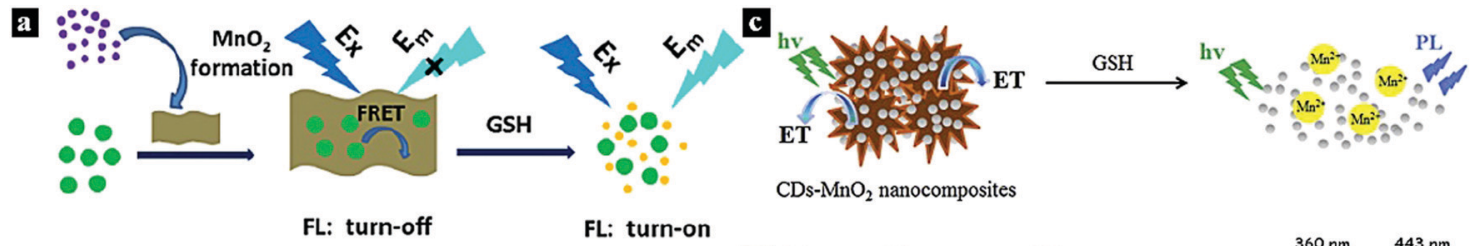

$\mathrm{CDs}-\mathrm{MnO}_{2}$ nanocomposites

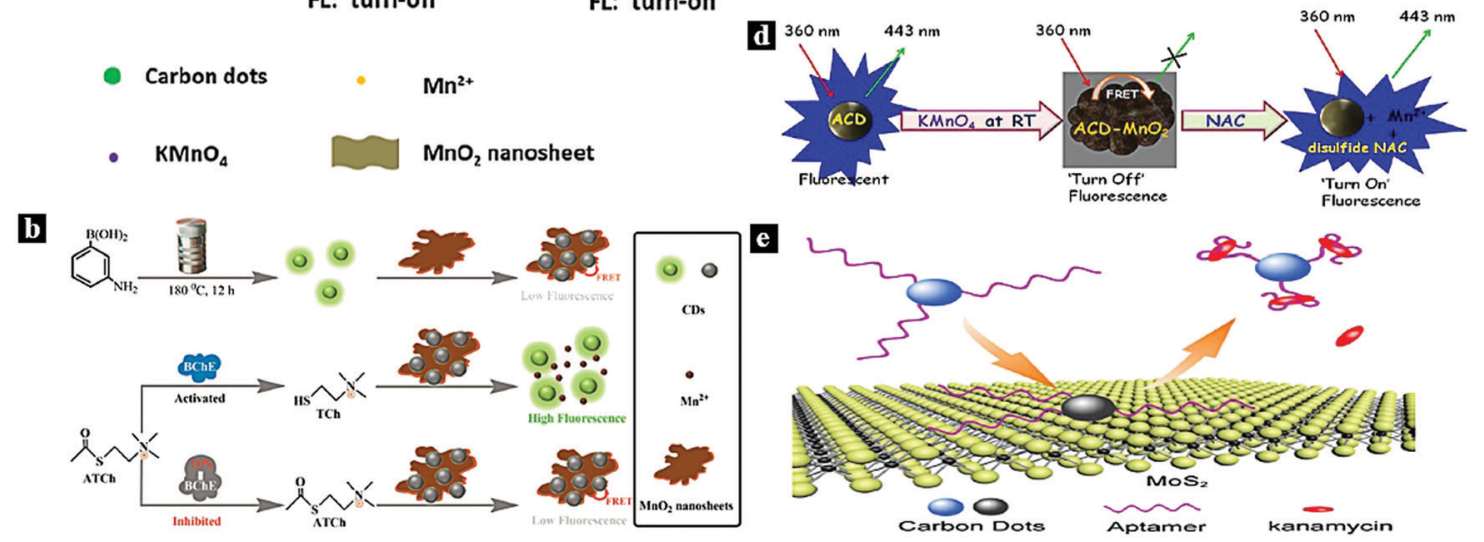

Fig. 6 (a) Schematic illustration of the preparation of a nanoprobe for GSH based on a CDots-MnO $\mathrm{O}_{2}$ FRET system. ${ }^{11}$ Reprinted with permission ref. 11 . Copyright 2015 The Royal Society of Chemistry. (b) Schematic illustration of a nanoprobe for organophosphorus pesticides based on a CDots-MnO 2 FRET system. ${ }^{74}$ Reprinted with permission ref. 70. Copyright 2018 American Chemical Society. (c) Schematic illustration of a nanoprobe for GSH based on a CDots $-\mathrm{MnO}_{2}$ FRET system. ${ }^{75}$ Reprinted with permission ref. 71 . Copyright 2015 Elsevier. (d) Schematic illustration of an NAC nanoprobe based on a FRET system. ${ }^{77}$ (e) Schematic illustration of a kanamycin sensing strategy based on a CDots-MoS 2 FRET system. ${ }^{81}$ Reprinted with permission ref. 73. Copyright 2017 Springer-Verlag Wien.

$\mathrm{MnO}_{2}$, resulting in fluorescence quenching; the fluorescence can be restored by adding NAC. ${ }^{77}$ In 2017 , Wang et al. synthesized a CDots- $\mathrm{MoS}_{2}$ FRET platform and used it for the sensitive detection of the antibiotic kanamycin (Fig. 6e). Through van der Waals forces, the CDots can be assembled onto the $\mathrm{MoS}_{2}$ surface, thus quenching the fluorescence due to the FRET from the CDots to $\mathrm{MoS}_{2} \cdot{ }^{81}$

\subsection{CDots \& Au/Ag}

Metal nanoparticles and particularly $\mathrm{Ag}$ and Au have attracted great research interest due to their physicochemical and photoelectric properties. Therefore, they are widely used in catalytic, photovoltaic, and biological applications. $\mathrm{Au}$ and $\mathrm{Ag}$ nanoparticles (AuNPs and AgNPs) can also be used as efficient acceptors for most fluorescent groups due to their high extinction coefficients and absorption bandwidths. Therefore, AuNPs ${ }^{89-95}$ and $\mathrm{AgNPs}^{96-100}$ are ideal nano-quenching agents to establish FRET-based nanoprobes due to their large extinction coefficients and wide absorption spectra, which overlap with the fluorescent emission of CDots.

In 2017 , Shen $e t$ al. synthesized a fluorescent nanoprobe for the sensitive detection of adenosine based on FRET. Fig. 7a schematically illustrates the nanoprobe for adenosine based on FRET between CDots and AuNPs. Through the hybridization between single-strand DNA (ssDNA)-functionalized CDots and aptamer-functionalized AuNPs, fluorescence quenching was observed due to FRET from the CDots to the AuNPs. When adenosine was present, the binding between adenosine and aptamer released the ssDNA-functionalized CDots from the AuNP surfaces, resulting in fluorescence recovery. ${ }^{84}$ In 2017,
$\mathrm{Li}$ et al. reported a CDots-Au nanocluster FRET system combined via a carbodiimide-activated coupling reaction as a ratiometric fluorescence nanoprobe for the bioimaging of $\mathrm{H}_{2} \mathrm{O}_{2}$ (Fig. 7b). The synthesized CDots-Au system showed a dualemission fluorescent property with $40 \%$ FRET efficiency from the CDots to Au. Furthermore, the red emission of the CDots-Au system could be quenched by $\mathrm{H}_{2} \mathrm{O}_{2}$, while the blue emission was used as a reference signal to provide built-in correction. ${ }^{85}$ In 2018, Ge et al. synthesized a selective and sensitive nanoprobe for the antibiotic D-penicillamine (D-PA) based on a FRET system between CDots and AuNPs combined via electrostatic interaction (Fig. 7c). In this system, AuNPs act as a colorimetric indicator and a fluorescence quencher. The CDot fluorescence can be quenched through the FRET process from the CDots to the AuNPs. Moreover, the quenching effect induced by the AuNPs can be restored by adding acetylthiocholine and D-PA to the FRET system. ${ }^{86}$ In 2019, Wang et al. synthesized a CDots-AuNCs FRET-based ratiometric fluorescent nanoprobe for $\mathrm{Pb}^{2+} / \mathrm{Cu}^{2+}$ (Fig. $7 \mathrm{~d}$ ). The probe was simply prepared by mixing AuNCs and N-CDots in aqueous solution. The probe can interact selectively with $\mathrm{Pb}^{2+}$ and $\mathrm{Cu}^{2+}$, leading to a shift in the fluorescent signal. The system showed good detection sensitivity at 0.5 and $0.15 \mu \mathrm{M}$ for $\mathrm{Pb}^{2+}$ and $\mathrm{Cu}^{2+}$, respectively. ${ }^{16}$ In 2018 , He et al. reported a CDots-AuNCs FRETbased dual-emission ratiometric fluorescence nanoprobe for dopamine sensing (Fig. 7e). The FRET-based nanoprobe consists of two fluorophores (AuNCs as the acceptor and CDots as the energy donor) and exhibits dual emission wavelengths of 610 and $420 \mathrm{~nm}$ under excitation at $380 \mathrm{~nm}$. The AuNCs were subsequently conjugated to the CDots via simple surface functionalization. 

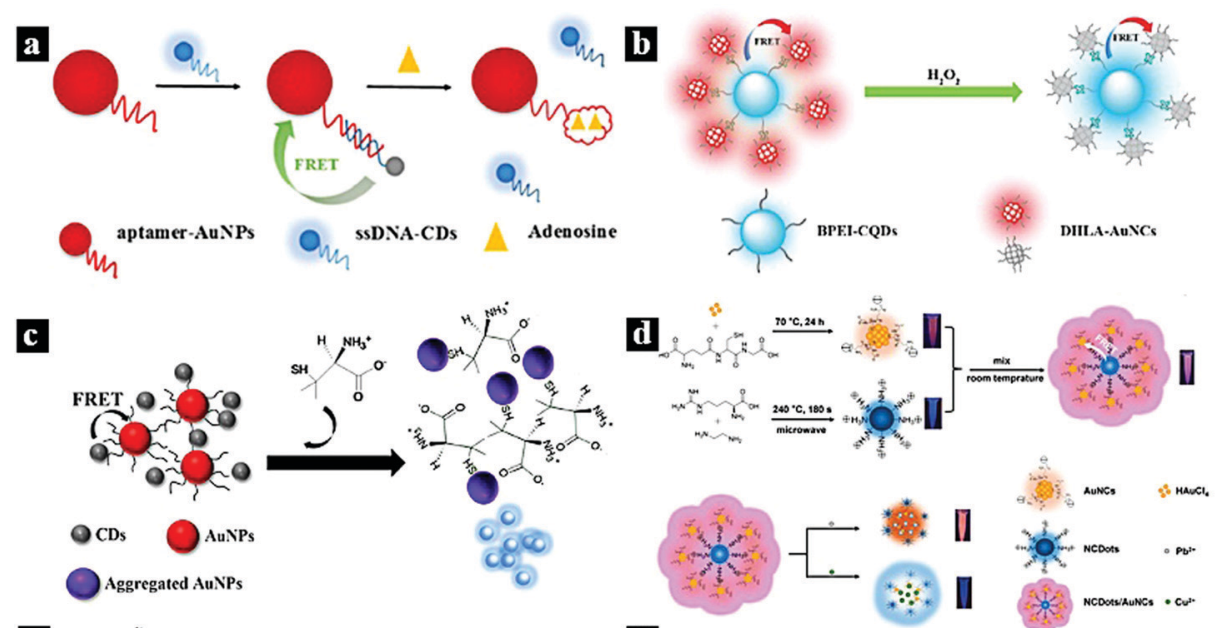

e

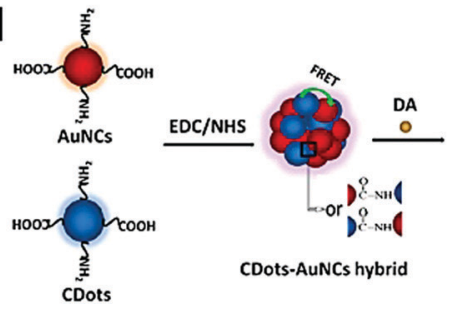

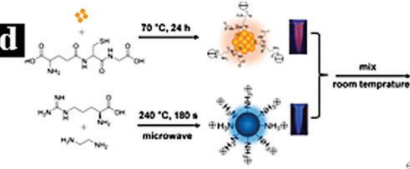

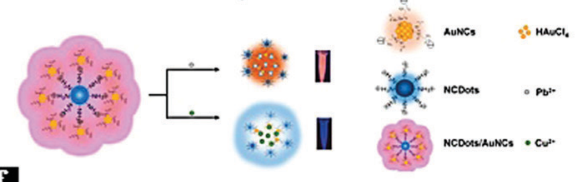

f


Fig. 7 (a) Schematic illustration of a nanoprobe for adenosine based on FRET between CDots and AuNPs. ${ }^{84}$ Reprinted with permission ref. 84. Copyright 2017 The Royal Society of Chemistry. (b) Schematic illustration of a FRET-based nanoprobe for $\mathrm{H}_{2} \mathrm{O}_{2} \cdot{ }^{85}$ Reprinted with permission ref. 85 . Copyright 2017 Elsevier. (c) Schematic illustration of a FRET-based nanoprobe for D-PA. ${ }^{86}$ Reprinted with permission ref. 86. Copyright 2018 Springer New York. (d) Schematic illustration of a ratiometric nanoprobe for $\mathrm{Pb}^{2+} / \mathrm{Cu}^{2+}$ based on a CDots-Au FRET system. ${ }^{16}$ Reprinted with permission ref. 16 . Copyright 2019 Elsevier. (e) Schematic illustration of a dopamine nanoprobe based on a CDots-Au FRET system. ${ }^{87}$ Reprinted with permission ref. 87. Copyright 2018 Elsevier. (f) Schematic illustration of a nanoprobe for L-cysteine based on a FRET system between CDots and AuNPs. ${ }^{88}$ Reprinted with permission ref. 88. Copyright 2019 Elsevier.

The fluorescence of the AuNCs can be quenched by dopamine, inhibiting the FRET process from the CDots to $\mathrm{Au}^{87}$ In 2019, Dong et al. reported a nanoprobe for the ratiometric detection of L-cysteine based on FRET between CDots and AuNPs (Fig. 7f). FRET was established from the energy donor (CDots) to the acceptor (AuNPs), which were combined via electrostatic interaction, effectively inhibiting the fluorescence emission of the CDots. After the addition of $\mathrm{L}$-cysteine to the system, the fluorescence of the NAC-AuNPs was quenched, while the emission intensity of the CDots remained almost constant. ${ }^{88}$

\subsection{CDots \& graphene oxide}

Graphene oxide (GO) is a two-dimensional graphitic carbon system with monoatomic thickness and oxygen-containing functional groups. Due to its good water solubility, large specific surface area, high electronic conductivity, and long-range nanoscale energy transfer characteristics, GO has attracted significant research attention. Recently, it has been used as a good energy acceptor to quench the fluorescence of CDots based on the FRET process. ${ }^{12,28,101-104}$

In 2012, Wei et al. reported a metal-ion nanoprobe with high selectivity and tunable dynamic range based on FRET from CDots to GO. Fig. 8a shows a schematic illustration of the nanoprobe for $\mathrm{K}^{+}$sensing based on the CDots-GO FRET system, in which the CDots and GO are combined via a specific cation-ligand complexation reaction. The fluorescence of the
CDots can be quenched by GO based on the FRET process; however, after the introduction of $\mathrm{K}^{+}$, which competes with ammonium functionalized on GO, the fluorescence of the CDots was restored. ${ }^{28}$ In 2016, Wu et al. synthesized a photoluminescent nanoprobe for the detection of lysozyme based on FRET from CDots to GO (Fig. 8b). Upon the addition of GO, the photoluminescence of the aptamer-conjugated CDots was effectively quenched because of the FRET from CDots to GO. However, in the presence of the target lysozyme, the CDots bound strongly to lysozyme via the aptamer-lysozyme interaction and desorbed from the GO surface, causing the photoluminescence of the CDots to recover. ${ }^{12}$ In 2015, Cui et al. synthesized a fluorescent nanoprobe for $\mathrm{Hg}^{2+}$ detection based on FRET between CDots and graphene oxide (Fig. 8c). The fluorescent signal of the CDots was quenched upon binding to GO based on the FERT process. However, when $\mathrm{Hg}^{2+}$ was present, oligodeoxyribonucleotide-functionalized CDots selectively bound to $\mathrm{Hg}^{2+}$ ions in solution and initiated the adsorption of CDots from GO, causing the CDot fluorescence to recover. ${ }^{101}$ In 2018, Cheng et al. synthesized a fluorometric nanoprobe for adenosine triphosphate (ATP) sensing based on FRET between CDots and GO (Fig. 8d). The CDots and GO were combined through $\pi$ stacking and hydrophobic interactions. CDots and GO acted as the energy donor and acceptor, respectively, resulting the quenching of the CDot fluorescence. When ATP was present, the aptamer bound strongly to ATP, causing 


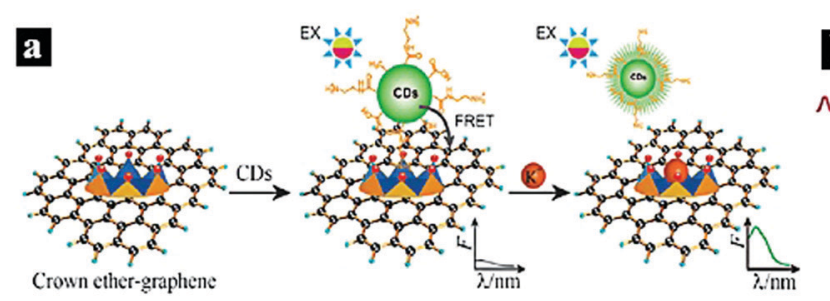

c

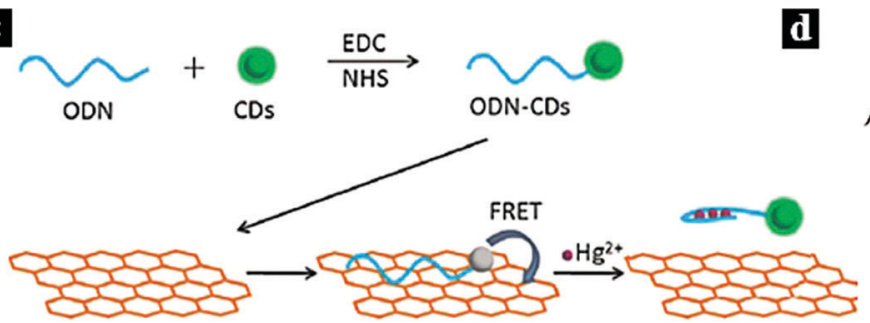

GO

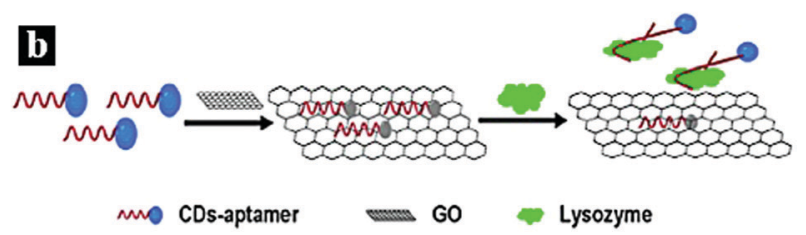

mo CDs-aptamer GO Lysozyme

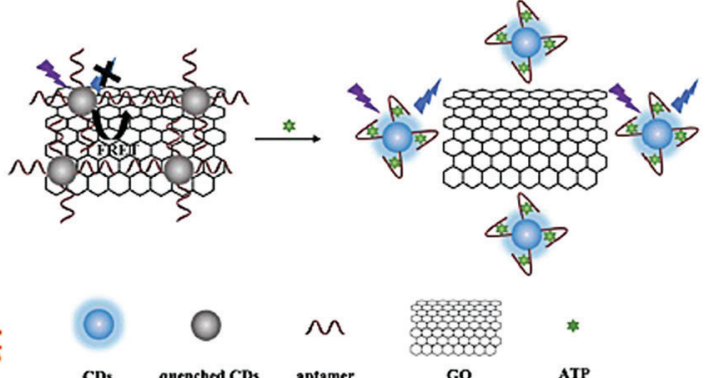

Fig. 8 (a) Schematic illustration of a nanoprobe for $\mathrm{K}^{+}$based on a CDots-GO FRET system. ${ }^{28}$ Reprinted with permission ref. 28 . Copyright 2012 The Royal Society of Chemistry. (b) Schematic illustration of a nanoprobe for lysozyme based on a CDots-GO FRET system. ${ }^{12}$ Reprinted with permission ref. 12. Copyright 2016 John Wiley and Sons Ltd. (c) Schematic illustration of a FRET system for $\mathrm{Hg}^{2+}$ detection. ${ }^{101}$ Reprinted with permission ref. 101. Copyright 2015 Elsevier Ltd. (d) Schematic illustration of a nanoprobe for ATP based on FRET between CDots and GO. ${ }^{102}$ Reprinted with permission ref. 102. Copyright 2018 Springer-Verlag Wien.

the CDots to unbind from GO and the CDot fluorescence to recover. $^{102}$

\subsection{CDots \& quantum dots}

Fluorescence resonance energy transfer between CDots and quantum dots has also been widely studied. The main studied quantum dots include CdTe, ${ }^{105}$ CDots, ${ }^{106}$ graphene quantum dots. ${ }^{107}$ In 2014, Tao et al. reported a nanoprobe for chlortoluron detection in water based on a FRET system between CDots and CdTe quantum dots. The FRET occurs from the CDots (donor) to CdTe (acceptor), greatly enhancing the fluorescence intensity of CdTe. After the addition of chlortoluron, the strong interaction between CdTe and chlortoluron leads to the quenching of CdTe fluorescence by the formation of the chlortoluron-CdTe ground state complex. ${ }^{105}$ In 2016, Wang et al. reported a nanoprobe for volatile organic compounds based on intra-particle FRET in Mn-CDots. The FRET occurs from the

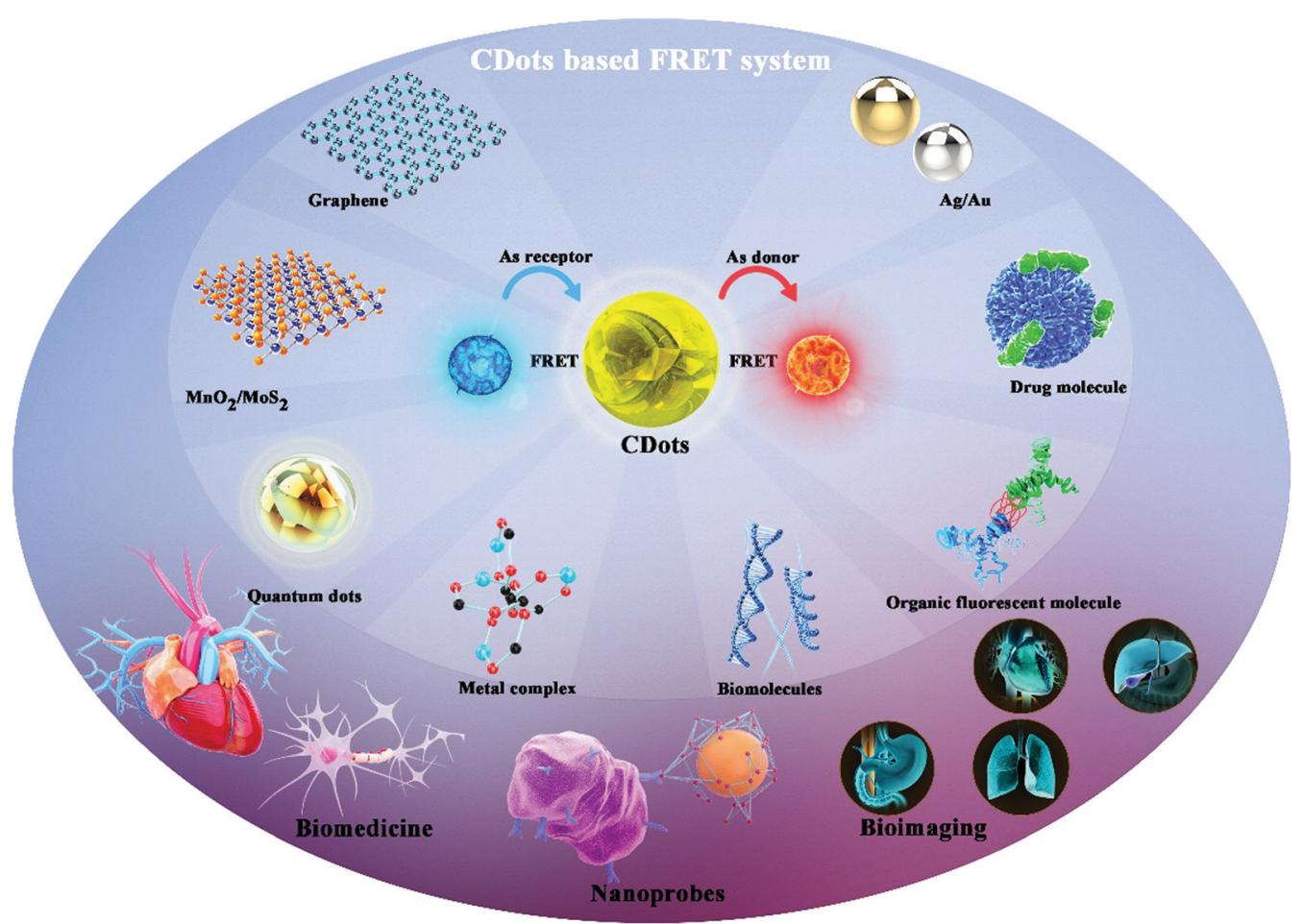

Fig. 9 Perspectives for potential future applications of Cdot-based FRET systems. 
surface-related energy donor to the metal-related energy acceptor in the Mn-CDots. The FRET is not sensitive to proximity; instead, it is sensitive to polarity resulting from the inherent fixed position of the two fluorescent groups on the CDot backbone. Therefore, based on the sensitivity of the FRET, the Mn-CDots have extraordinary sensing ability for some small molecules. ${ }^{106}$ In 2019 , Chini et al. reported a nanoprobe for sensing heavy metal ions based on a FRET system between CDots and graphene quantum dots. In this FRET system, the graphene quantum dots serve as the energy donor, while the CDots are the acceptor. After the addition of heavy metals such as mercury $\left(\mathrm{Hg}^{2+}\right)$ and arsenic $\left(\mathrm{As}^{5+}\right)$ into this nanoprobe system, the FRET signal was significantly reduced. ${ }^{107}$

\section{Conclusions \& perspectives}

CDots have recently emerged as a new class of fluorescent carbon nanomaterials and gained tremendous attention due to their excellent physicochemical properties, which distinguish them from traditional fluorescent materials. The major advantages of Cdot-based FRET systems include high sensitivity, adjustable composition, and good biocompatibility, which make them promising materials for nanoprobes, bioimaging, and biomedicine. In comparison with conventional FRET systems, CDot-based FRET systems overcome drawbacks such as spectral crosstalk, photobleaching, and direct acceptor excitation. CDot-based FRET systems also provide increased sensitivity, for single function and multifunction use. As a new type of nanomaterial with excellent optical properties, CDots can serve as an energy donor and receptor, greatly improving the application range and efficacy of CDot-based FRET systems. Perspectives for potential future applications of CDot-based FRET systems are summarized in Fig. 9.

Research on the design and fabrication of CDot-based FRET systems has become of great interest in chemical and biological sciences along with engineering fields. With the development of FRET-based nanoprobes, the functional units of FRET have been expanded from simple molecules to macromolecule and nanoparticles, and the research focus has shifted from single measurements to multifunctional and interdisciplinary applications. CDot-based FRET nanoprobes show promise for achieving high sensitivity, high selectivity, portability, fast response, and biocompatibility, which are important in applications in biotechnology, the environment, and medicine. For example, all-in-one diagnostic platforms for recognition, imaging, and therapy have received increasing attention. However, a great number of challenges and opportunities remain. ${ }^{108}$ For example, while numerous optical light-emitting diode devices have been reported, further research in the field of biological devices is needed. This review summarized recent progress in the research and development of CDot-based FRET systems with a focus on the different classifications of fluorescent molecules and potential applications in nanoprobes. We reviewed several fluorescent molecules in detail, including organic fluorescent molecules, drug molecules, metal complexes, biomolecules, $\mathrm{MnO}_{2} / \mathrm{MoS}_{2}, \mathrm{Au} / \mathrm{Ag}$, graphene/GO, and quantum dots. We also presented extensive applications of CDot-based FRET systems as highly sensitive nanoprobes for diverse analytes.

Although many applications of CDot-based FRET systems have been reported, challenges remain. First, for CDots acting as the energy donor, new energy receptors are needed to facilitate efficient energy transfer and increase detection sensitivity. Second, for CDots acting as the energy receptor, new energy donors should be explored. Thus, CDots can be enabled to exhibit superior optical performance for nanoprobe and biological applications. Third, novel CDots based on heteroatom doping and surface functionalization should be explored to allow the FRET process to be modulated by preparing CDots with different properties. In summary, exploring new energy transfer donor and receptor pairs for FRET systems is important to improve FRET efficiency and analytical performance. CDotbased FRET systems will undoubtedly overcome the limitations of traditional luminescent materials and have a bright future in nanoprobes, bioimaging, biomedicine and other applications.

\section{Conflicts of interest}

There are no conflicts to declare.

\section{Acknowledgements}

This work was supported by the National Key R\&D Program of China (2017YFA0206901, 2017YFA0206900), the NSF of China (21705027), the Major Scientific and Technological Innovation Project of Shandong (2017CXGC0501), the Natural Science Foundation of Shandong Province (ZR2017PEM004, and ZR2017BEM020), the Recruitment Program of Global Experts of China and the Recruitment Program of Global Experts of Shanghai, and the Australia National Health and Medical Research Council (NHMRC APP1163786).

\section{References}

1 A. T. Aron, M. O. Loehr, J. Bogena and C. J. Chang, J. Am. Chem. Soc., 2016, 138, 14338-14346.

2 R. Vafabakhsh, J. Levitz and E. Y. Isacoff, Nature, 2015, 524, 497.

3 J. Guo, X. Qiu, C. Mingoes, J. R. Deschamps, K. Susumu, I. L. Medintz and N. Hildebrandt, ACS Nano, 2019, 13, 505-514.

4 J. Liu and Y. Lu, J. Am. Chem. Soc., 2002, 124, 15208-15216.

5 E. Galperin, V. V. Verkhusha and A. Sorkin, Nat. Methods, 2004, 1, 209-217.

6 Y. Yang, H. Liu, M. Han, B. Sun and J. Li, Angew. Chem., Int. Ed., 2016, 55, 13538-13543.

7 S. Basu, L.-M. Needham, D. Lando, E. J. R. Taylor, K. J. Wohlfahrt, D. Shah, W. Boucher, Y. L. Tan, L. E. Bates, O. Tkachenko, J. Cramard, B. C. Lagerholm, C. Eggeling, B. Hendrich, D. Klenerman, S. F. Lee and E. D. Laue, Nat. Commun., 2018, 9, 2520. 
8 H. Peng, L. Jing, W. Xiansong, W. Zhe, Z. Chunlei, H. Meng, W. Kan, C. Feng, L. Zhiming and S. Guangxia, Adv. Mater., 2012, 24, 5104-5110.

9 T. Jing, K. Biao, W. Hao, X. Ming, W. Yongcheng, W. Yanli, Z. Dongyuan and Z. Gengfeng, Adv. Mater., 2013, 25, 6569-6574.

10 F. Du, Y. Min, F. Zeng, C. Yu and S. Wu, Small, 2014, 10, 964-972.

11 W. Yuhui, J. Kai, Z. Jiali, Z. Ling and L. Hengwei, Chem. Commun., 2015, 51, 12748-12751.

12 J. Wu, Y. Hou, P. Wang, Z. Wang, Y. Li, S. Wang and M. Yang, Luminescence, 2016, 31, 1207-1212.

13 Z. Liang, M. Kang, G. F. Payne, X. Wang and R. Sun, ACS Appl. Mater. Interfaces, 2016, 8, 17478-17488.

14 C. Liu, D. Ning, C. Zhang, Z. Liu, R. Zhang, J. Zhao, T. Zhao, B. Liu and Z. Zhang, ACS Appl. Mater. Interfaces, 2017, 9, 18897-18903.

15 A. Chatzimarkou, T. G. Chatzimitakos, A. Kasouni, L. Sygellou, A. Avgeropoulos and C. D. Stalikas, Sens. Actuators, B, 2018, 258, 1152-1160.

16 L. Wang, H.-X. Cao, Y.-S. He, C.-G. Pan, T.-K. Sun, X.-Y. Zhang, C.-Y. Wang and G.-X. Liang, Sens. Actuators, B, 2019, 282, 78-84.

17 G. Chen, F. Song, X. Xiong and X. Peng, Ind. Eng. Chem. Res., 2013, 52, 11228-11245.

18 B. Prevo and E. J. G. Peterman, Chem. Soc. Rev., 2014, 43, 1144-1155.

19 W. R. Algar, H. Kim, I. L. Medintz and N. Hildebrandt, Coord. Chem. Rev., 2014, 263-264, 65-85.

20 J. Tang, Y. Zhang, B. Kong, Y. Wang, P. Da, J. Li, A. A. Elzatahry, D. Zhao, X. Gong and G. Zheng, Nano Lett., 2014, 14, 2702-2708.

21 B. Kong, J. Tang, Y. Zhang, T. Jiang, X. Gong, C. Peng, J. Wei, J. Yang, Y. Wang, X. Wang, G. Zheng, C. Selomulya and D. Zhao, Nat. Chem., 2015, 8, 171.

22 S. Tao, S. Lu, Y. Geng, S. Zhu, S. A. T. Redfern, Y. Song, T. Feng, W. Xu and B. Yang, Angew. Chem., Int. Ed., 2018, 57, 2393-2398.

23 H. Li, Z. Kang, Y. Liu and S.-T. Lee, J. Mater. Chem., 2012, 22, 24230-24253.

24 L. Wang, X. Wu, S. Guo, M. Han, Y. Zhou, Y. Sun, H. Huang, Y. Liu and Z. Kang, J. Mater. Chem. A, 2017, 5, 2717-2723.

25 B. Kong, A. Zhu, C. Ding, X. Zhao, B. Li and Y. Tian, Adv. Mater., 2012, 24, 5844-5848.

26 X. Xu, R. Ray, Y. Gu, H. J. Ploehn, L. Gearheart, K. Raker and W. A. Scrivens, J. Am. Chem. Soc., 2004, 126, 12736-12737.

27 Y.-P. Sun, B. Zhou, Y. Lin, W. Wang, K. A. S. Fernando, P. Pathak, M. J. Meziani, B. A. Harruff, X. Wang, H. Wang, P. G. Luo, H. Yang, M. E. Kose, B. Chen, L. M. Veca and S.-Y. Xie, J. Am. Chem. Soc., 2006, 128, 7756-7757.

28 W. Wei, C. Xu, J. Ren, B. Xu and X. Qu, Chem. Commun., 2012, 48, 1284-1286.

29 S. Yu, Y. L. Su, H. N. Umh and J. Yi, Nano Energy, 2016, 26, 479-487.
30 Y. Su, S. Lu, P. Gao, M. Zheng and Z. Xie, Mater. Chem. Front, 2019, 3, 1747-1753.

31 Y. Changmin, L. Xizhen, Z. Fang, Z. Fangyuan and W. Shuizhu, Chem. Commun., 2013, 49, 403-405.

32 C. Yu, Y. Wu, Z. Fang and S. Wu, J. Mater. Chem. B, 2013, 1, 4152.

33 F. Du, Y. Ming, F. Zeng, C. Yu and S. Wu, Nanotechnology, 2013, 24, 365101.

34 N. Sukhendu, M. Ravit, P. K. Kaviya, M. Yelena, K. Sofiya and J. Raz, Chem. Commun., 2014, 50, 10299-10302.

$35 \mathrm{~S} . \mathrm{Hu}, \mathrm{Q}$. Zhao, Q. Chang, J. Yang and J. Liu, RSC Adv., 2014, 4, 41069-41075.

36 D. Shi, F. Yan, T. Zheng, Y. Wang, X. Zhou and C. Li, RSC Adv, 2015, 5, 98492-98499.

37 S. Nandi, R. Malishev, S. K. Bhunia, S. Kolusheva, J. Jopp and R. Jelinek, Biophys. J., 2016, 110, 2016-2025.

38 K. Yang, L. Fan, W. Y. Che, X. Hu and T. Feng, RSC Adv., 2016, 6, 101447-101451.

39 M. Deng, W. Sha, C. Liang, H. Shang and S. Jiang, RSC Adv., 2016, 6, 26936-26940.

40 J. Hou, Z. Tian, H. Xie, Q. Tian and S. Ai, Sens. Actuators, B, 2016, 232, 477-483.

41 J. S. Sidhu, A. Singh, N. Garg and N. Singh, ACS Appl. Mater. Interfaces, 2017, 9, 25847-25856.

42 J. Kudr, L. Richtera, K. Xhaxhiu, D. Hynek, Z. Heger, O. Zitka and V. Adam, Biosens. Bioelectron., 2017, 92, 133-139.

43 Y. Yuan, J. Jiang, S. Liu, J. Yang, Z. Hui, J. Yan and X. Hu, Sens. Actuators, B, 2017, 242, 545-553.

44 H. Wang, Q. Lu, Y. Liu, H. Li, Y. Zhang and S. Yao, Sens. Actuators, B, 2017, 250, 429-435.

45 H. Chao-Peng, H. Zahralsaadat, A. Efe, Z. Shanyu, S. Michel, Z. Haijiang, G. Huizhang, W. Lukas, R. M. Rossi and M. M. Koebel, Sens. Actuators, B, 2017, 253, 714-722.

46 H. Liu, C. Xu, Y. Bai, L. Liu, D. Liao, J. Liang, L. Liu and H. Han, Spectrochim. Acta, Part A, 2017, 171, 311-316.

47 Y. Xia, L. Wang, J. Li, X. Chen and J. H. Chen, Anal. Chem., 2018, 90, 8969-8976.

48 J. S. Sidhu and N. Singh, J. Mater. Chem. B, 2018, 6, 4139-4145.

49 A. Das, D. Roy, M. Mandal, C. Jaiswal, M. Ta and P. K. Mandal, J. Phys. Chem. Lett., 2018, 9, 5092-5099.

50 S. Kumari, M. Tiwari and P. Das, Sens. Actuators, B, 2019, 279, 393-399.

51 Y. Shu, N. Zheng, A.-Q. Zheng, T.-T. Guo, Y.-L. Yu and J.-H. Wang, Anal. Chem., 2019, 91, 4157-4163.

52 J. S. Sidhu, A. Singh, N. Garg, N. Kaur and N. Singh, Sens. Actuators, B, 2019, 282, 515-522.

53 J. Wang, J. Wei, S. Su and J. Qiu, New J. Chem., 2015, 39, 501-507.

54 T. Feng, H. J. Chua and Y. Zhao, ACS Biomater. Sci. Eng., 2017, 3, 1535-1541.

55 J. Wang, S. Su, J. Wei, R. Bahgi, L. Hope-Weeks, J. Qiu and S. Wang, Physica E, 2015, 72, 17-24.

56 H. Chen, Z. Wang, S. Zong, P. Chen, D. Zhu, L. Wu and Y. Cui, Nanoscale, 2015, 7, 15477-15486.

57 S. Liu, IOP Conf. Ser., 2018, 382, 022055. 
58 F. Colin, N. Nikolitsa, A. P. Mchale, M. C. Bridgeen and J. F. Callan, Chem. Commun., 2013, 49, 8934-8936.

59 J. Wang, Z. Zhang, S. Zha, Y. Zhu, P. Wu, B. Ehrenberg and J. Y. Chen, Biomaterials, 2014, 35, 9372-9381.

60 L. Lin, Y. Wang, Y. Xiao and X. Chen, Anal. Bioanal. Chem., 2019, 411, 2803-2808.

61 Y. Shi, C. Li, S. Liu, Z. Liu, J. Zhu, J. Yang and X. Hu, RSC Adv, 2015, 5, 64790-64796.

62 M. Ganiga and J. Cyriac, Sens. Actuators, B, 2016, 225, 522-528.

63 Y. Kim, G. Jang and T. S. Lee, ACS Appl. Mater. Interfaces, 2015, 7, 15649-15657.

64 L. Linbo, W. Chao, L. Kangyu, W. Yuhan, L. Kun and L. Yuqing, Anal. Chem., 2015, 87, 3404-3411.

65 G. Li, W. Kong, M. Zhao, S. Lu, P. Gong, G. Chen, L. Xia, H. Wang, J. You and Y. Wu, Biosens. Bioelectron., 2016, 79, 728-735.

66 M. Yang, W. Kong, L. Hao, J. Liu, H. Hui, L. Yang and Z. Kang, Micro. Acta, 2015, 182, 2443-2450.

67 J. Chen, Y. Li, K. Lv, W. Zhong, H. Wang, Z. Wu, P. Yi and J. Jiang, Sens. Actuators, B, 2016, 224, 298-306.

68 D. Yang, S. Guan, Y. Niu, Z. Xie, S. Zhou and X. Qu, J. Mater. Chem. B, 2018, 6, 2315-2322.

69 F. Yan, Z. Bai, Y. Chen, F. Zu, X. Li, J. Xu and L. Chen, Sens. Actuators, B, 2018, 275, 86-94.

70 F. Khakbaz and M. Mahani, Anal. Biochem., 2017, 523, 32-38.

71 Y. Q. Dang, Y. J. Zhou, J. T. Cai, G. Y. Liu, Y. T. Zhang and J. S. Qiu, J. Nano Res., 2017, 45, 134-141.

72 T. Zhang, H. Xu, W. He, J. Zhu, Z. Yue, B. Xue, B. Dong and H. Song, RSC Adv, 2017, 7, 28987-28993.

73 Q. Y. Cai, J. Li, J. Ge, L. Zhang, Y. L. Hu, Z. H. Li and L. B. Qu, Biosens. Bioelectron., 2015, 72, 31-36.

74 X. Yan, Y. Song, C. Zhu, H. Li, D. Du, X. Su and Y. Lin, Anal. Chem., 2018, 90, 2618-2624.

75 C. Yang, W. Deng, H. Liu, S. Ge and Y. Mei, Sens. Actuators, $B, 2015,216,286-292$.

76 Y. Hu, L. Zhang, X. Geng, J. Ge, H. Liu and Z. Li, Anal. Methods, 2017, 9, 5653-5658.

77 J. Jana, T. Aditya, M. Ganguly and T. Pal, Sens. Actuators, B, 2017, 246, 716-725.

78 J. Yang, Z. Huang, Y. Hu, J. Ge, J. Li and Z. Li, New J. Chem., 2018, 42, 15121-15126.

79 D. Garg, A. Mehta, A. Mishra and S. Basu, Spectrochim. Acta, Part A, 2018, 192, 411-419.

80 K. Srinivasan, K. Subramanian, K. Murugan and K. Dinakaran, Analyst, 2016, 141, 6344-6352.

81 Y. Wang, T. Ma, S. Ma, Y. Liu, Y. Tian, R. Wang, Y. Jiang, D. Hou and J. Wang, Micro. Acta, 2017, 184, 203-210.

82 S. Hamd-Ghadareh, A. Salimi, S. Parsa and F. Fathi, Int. J. Biol. Macromol., 2018, 118, 617-628.

83 S. Gogoi and R. Khan, Phys. Chem. Chem. Phys., 2018, 20, 16501-16509.
84 X. Shen, L. Xu, W. Zhu, B. Li, J. Hong and X. Zhou, New J. Chem., 2017, 41, 9230-9235.

85 Z. Li, G. Song, Z. Yuan and L. Chao, Sens. Actuators, B, 2017, 241, 821-827.

86 H. Ge, K. Zhang, H. Yu, J. Yue, L. Yu, X. Chen, T. Hou, K. A. Alamry, H. M. Marwani and S. Wang, J. Fluoresc., 2018, 28, 1405-1412.

87 Y.-S. He, C.-G. Pan, H.-X. Cao, M.-Z. Yue, L. Wang and G.-X. Liang, Sens. Actuators, B, 2018, 265, 371-377.

88 W. Dong, R. Wang, X. Gong, W. Liang and C. Dong, Spectrochim. Acta, Part A, 2019, 213, 90-96.

89 Y. Shi, Y. Pan, H. Zhang, Z. Zhang, M.-J. Li, C. Yi and M. Yang, Biosens. Bioelectron., 2014, 56, 39-45.

90 H. Dai, S. Yan, Y. Wang, Y. Sun, J. Hu, P. Ni and L. Zhuang, Sens. Actuators, B, 2014, 202, 201-208.

91 M. Xu, Z. Gao, Z. Qian, Y. Lin, M. Lu and D. Tang, Biosens. Bioelectron., 2016, 86, 978-984.

92 X. Wu, Y. Song, X. Yan, C. Zhu, Y. Ma, D. Du and Y. Lin, Biosens. Bioelectron., 2017, 94, 292.

93 J. Korram, L. Dewangan, R. Nagwanshi, I. Karbhal, K. K. Ghosh and M. L. Satnami, New J. Chem., 2019, 43, 6874-6882.

94 S. Xu, F. Zhang, L. Xu, X. Liu, P. Ma, Y. Sun, X. Wang and D. Song, Sens. Actuators, B, 2018, 273, 1015-1021.

95 Y. Yang, D. Huo, H. Wu, X. Wang, J. Yang, M. Bian, Y. Ma and C. Hou, Sens. Actuators, B, 2018, 274, 296-303.

96 M. Amjadi, Z. Abolghasemi-Fakhri and T. Hallaj, J. Photochem. Photobiol., A, 2015, 309, 8-14.

97 P. A. Sajid, S. S. Chetty, S. Praneetha, A. V. Murugan, Y. Kumar and L. Periyasamy, RSC Adv., 2016, 6, 103482-103490.

98 S. Li, J. Wang, W. Sheng, W. Wen, Y. Gu and S. Wang, Micro. Acta, 2018, 185, 388.

99 C. Wang, R. Tan and D. Chen, Talanta, 2018, 182, 363-370.

100 J.-T. Cao, W.-S. Zhang, H. Wang, S.-H. Ma and Y.-M. Liu, New J. Chem., 2019, 43, 1424-1430.

101 X. Cui, L. Zhu, J. Wu, Y. Hou, P. Wang, Z. Wang and M. Yang, Biosens. Bioelectron., 2015, 63, 506-512.

102 X. Cheng, Y. Cen, G. Xu, F. Wei, M. Shi, X. Xu, M. Sohail and Q. Hu, Micro. Acta, 2018, 185, 144.

103 Y. Ding, J. Ling, H. Wang, J. Zou, K. Wang, X. Xiao and M. Yang, Anal. Methods, 2015, 7, 7792-7798.

104 X. Fu, L. Sheng, Y. Yu, M. Ma, Z. Cai and X. Huang, Sens. Actuators, B, 2018, 269, 278-287.

105 H. Tao, X. Liao, C. Sun, X. Xie, F. Zhong, Z. Yi and Y. Huang, Spectrochim. Acta, Part A, 2015, 136, 1328-1334.

106 Y. Wang, H. Meng, M. Jia, Y. Zhang, H. Li and L. Feng, Nanoscale, 2016, 8, 17190-17195.

107 M. K. Chini, V. Kumar, A. Javed and S. Satapathi, NanoStruct. Nano-Objects, 2019, 19, 100347.

108 J. Wang, F. Zhang, Y. Wang, Y. Yang and X. Liu, Carbon, 2018, 126, 426-436. 